\title{
Mobile Application for Impedance-Based Biomimetic Sensor Readout
}

\author{
Jeroen Broeders, Dieter Croux, Marloes Peeters, Thomas Beyens, Stijn Duchateau, Thomas J. Cleij, \\ Patrick Wagner, Ronald Thoelen, and Ward De Ceuninck
}

\begin{abstract}
A new method is presented for smartphone-based impedance spectroscopy, especially fine-tuned for biomimetic sensor readout. Complete user control is given by means of an app while the on-board audio hardware of the smartphone or tablet $\mathrm{PC}$ is used to perform impedance measurements. This considerably limits the required external hardware. Disposable test strips can be mounted for convenient readout of various sensors. The system is verified on passive components and a synthetic molecularly imprinted polymer histamine sensor. The prototype design could prove a useful step toward the development of home-diagnostics biosensing applications.
\end{abstract}

Index Terms-Biomimetic sensors, impedance spectroscopy, molecular imprinting, smartphone.

\section{INTRODUCTION}

B IOMIMETIC sensors offer huge potential in the development of point-of-care systems and home-diagnostics applications. Biological relevant molecules in an analyte can bind to a highly specific artificial bio-recognition layer, while this binding event is transduced to a measurable signal. The development of different types of these sensors suited for the detection of a wide range of target molecules is a popular field of research. A commercial biomimetic sensor market is almost nonexistent, though examples can be found of commercially available biosensing devices, e.g. the portable glucose sensor, which is already used for decades by diabetes patients, and digital pregnancy tests.

Electrochemical impedance spectroscopy is a popular readout technique commonly used for different types of

Manuscript received January 22, 2013; revised February 28, 2013; accepted March 27, 2013. Date of publication April 3, 2013; date of current version May 31, 2013. This work was supported in part by the Special Research Funds of Hasselt University and Xios University College. The associate editor coordinating the review of this paper and approving it for publication was Prof. Octavian Postolache.

J. Broeders and S. Duchateau are with the Institute for Materials Research, Hasselt University, Diepenbeek B-3590, Belgium, and also with Xios University College, Diepenbeek B-3590, Belgium (e-mail: jeroen.broeders@uhasselt.be; stijn.duchateau@xios.be).

D. Croux, M. Peeters, and T. J. Cleij are with the Institute for Materials Research, Hasselt University, Diepenbeek B-3590, Belgium (e-mail: dieter.croux@uhasselt.be; marloes.peeters@uhasselt.be; thomas.cleij@uhasselt.be).

T. Beyens and R. Thoelen are with Xios University College, Diepenbeek B-3590, Belgium (e-mail: thomas.beyens@student.xios.be; ronald.thoelen@ xios.be).

P. Wagner and W. De Ceuninck are with Hasselt University, Institute for Materials Research, Diepenbeek B-3590, Belgium, and also with IMEC, Division IMO-MEC, Diepenbeek B-3590, Belgium (e-mail: patrick.wagner@uhasselt.be; ward.deceuninck@uhasselt.be).

Color versions of one or more of the figures in this paper are available online at http://ieeexplore.iee.org.

Digital Object Identifier 10.1109/JSEN.2013.2256346 sensors [2], [3]. Within these sensors the impedimetric changes in the sensor/electrolyte interface upon target molecule binding are usually monitored using bulky and expensive labbased measurement equipment. The home-diagnostics market could benefit greatly from compact, easy-to-use devices that are suited for the readout of a wide range of sensors. Previous research resulted in the development of miniaturized impedimetric measurement equipment incorporating touch-screen technology and numerous communication protocols [4]. Although this device is specifically designed for accurate biosensor readout while maintaining a level of user-friendliness, it is not fine-tuned for home-monitoring applications.

Some examples can already be found of smartphones apps used in home diagnostics, e.g. for glucose level sensing [5] and in heart rate monitoring applications [6].

A novel way of impedimetric readout of biomimetic sensors via smartphones and tablet PCs is presented. On-board audio hardware is used for generating and reading signals, minimizing the required external hardware. A custom-made biosensor test strip can be inserted in the system. Small amounts of analyte can be placed on the strip, after which sensor response can be monitored. An app running on the device allows for intuitive control and graphical display of biosensor response. Device performance is verified using passive components and a biosensor example.

Molecularly imprinted polymer (MIP) sensors are a commonly used type of biomimetic sensors. The sensors are based on polymers with imprinted nano-cavities that form preferential binding sites for the desired target molecule. Using these sensors as synthetic receptors offers two main benefits in addition to specific recognition. First, MIPs can be synthesized at a rather low cost via established polymerchemical routes [7], [8]. Second, MIPs can be stored for long time scales and maintain their receptor properties in wide ranges of temperature, $\mathrm{pH}$, and ionic strength [9]-[11]. These sensors have already been proven to be extremely suited for the detection of various low molecular weight targets such as histamine [12], L-nicotine [13], [14] and adenine [15]. The newly developed system is demonstrated using a MIP-based histamine sensor.

\section{Materials AND MethodS}

\section{A. System Overview}

Impedimetric readout of biomimetic sensors and biosensors in general, is often performed within a frequency spectrum of $50 \mathrm{~Hz}$ to $10 \mathrm{kHz}$. The exact frequency is strongly dependent 
on the specific sensor purpose and target. Examples include a frequency of tens of Hertz for immunosensors [16], several hundred Hertz for MIPs [17] and $10 \mathrm{kHz}$ for DNA denaturation based sensors [18]. These frequencies lie well within the audio spectrum of $20 \mathrm{~Hz}$ to $20 \mathrm{kHz}$. The amplitude of the excitation voltages is usually kept well below $100 \mathrm{mV}$ to avoid interference with the biochemical reactions occurring in the measurement cell. Furthermore, when working toward biological cell-based sensors, the potential needs to be lower than $60 \mathrm{mV}$ to avoid the triggering of ion channels [19]. Last but not least, biosensor measurements are rarely performed over a single channel. It is usually required to measure at least one reference channel together with the sensor, to rule out side effect like temperature fluctuation and nonspecific binding events.

The standard audio outputs of smartphones, normally used for connecting headphones, are well suited for these potentials within the desired frequency range. The left and right audio channel can be used for two-channel signal output. After conversion to a voltage the resulting current caused by the impedance of a sample can be read-in by the microphone input of the device. Since both a 2-channel audio output and a microphone input are commonly present on the same 4-pole audio jack, this allows for miniature, user-friendly impedimetric biosensor readout setups.

It is a general consensus to differentiate between smartphones and tablet PCs based on the operating system (OS). Since the Apple Inc. proprietary iOS runs only on Apple Inc. devices, one has a large degree of certainty and uniformity in the hardware present in these devices. Therefore, as a first step in post-PC device biosensor readout, the choice was made to develop the system for the Darwin-based iOS, though the software could be ported to other operating systems if desired. The audio in- and output signals are completely controlled by an app, which also performs all necessary calculations and plotting of data and allows for user-friendly, intuitive control.

The maximum sample rate of the audio in/output of the chosen devices lies at $44.1 \mathrm{kHz}$ with a bit depth of 16 bit. This puts some limitations on the impedance and frequency resolution and range, which will be taken into account during design and performance verification.

\section{B. Hardware Implementation}

As excitation signal generation and reading the resulting voltages is completely done by respectively the audio output and microphone input of the smartphone, only a minimum of external hardware is required. The developed hardware is shown in Fig. 1, together with the circuit diagram.

The $50 \mathrm{mV}$ sine wave, alternately generated by the left and right channel audio output, is buffered by means of an Analog Devices AD8030 dual operational amplifier (OPAMP). This OPAMP has a wide operating voltage range with a relatively low power consumption of $1.3 \mathrm{~mA}$, making it suitable in low-power devices. The buffered signals of both channels are subsequently connected to either a test strip, mounted in a Samtec card edge connector, or to shielded coaxial cables to connect with existing lab equipment.
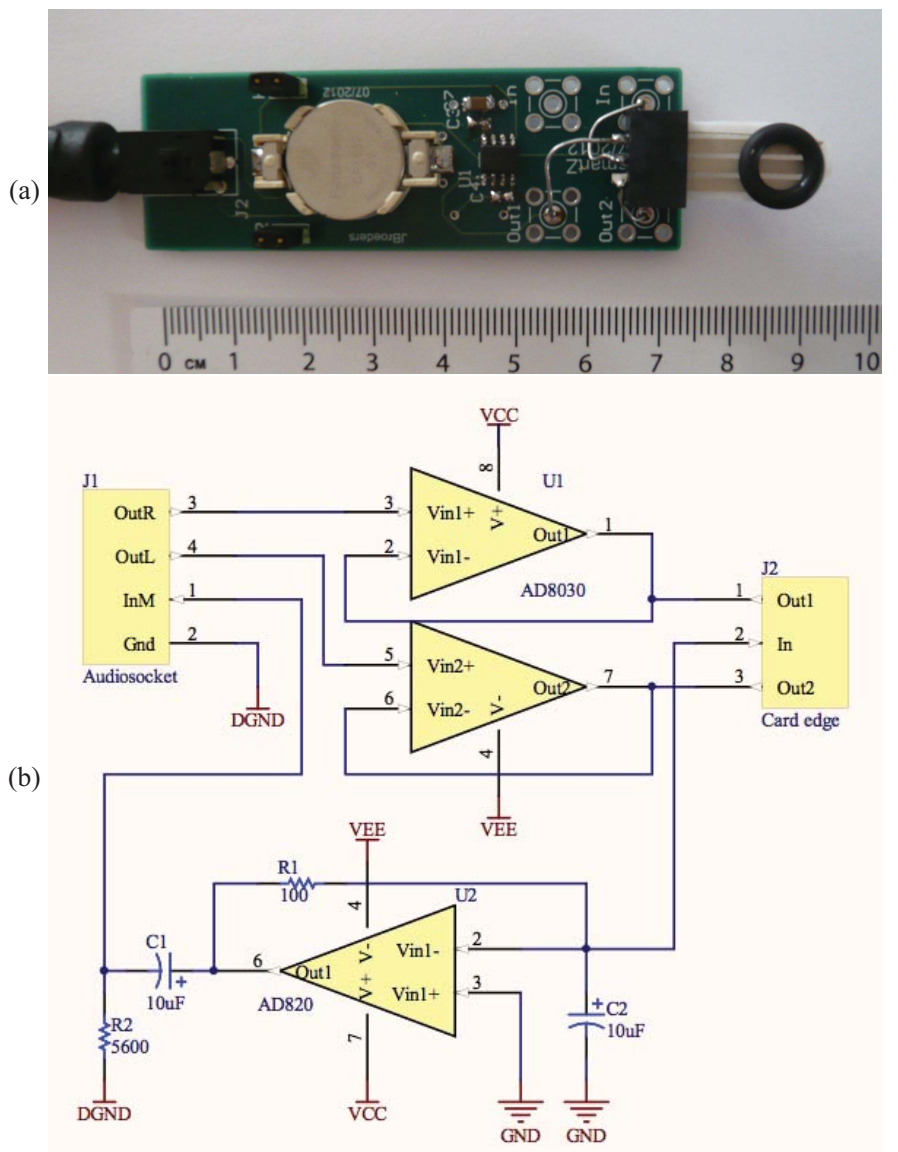

Fig. 1. (a) Hardware interfaced to the smartphone with test strip mounted and (b) circuit diagram.

An AD820 inverting OPAMP circuit converts the resulting current, resulting from the impedance of an attached sample, back to a voltage. This OPAMP was again chosen for its wide operating voltage and low quiescent current. The resulting voltage can be read by the microphone input of the smartphone, though it should be noted that this input is limited to about $42 \mathrm{mV}$. Since the samples behave like input resistors of the inverting OPAMP circuit, operating at a fixed input voltage of $50 \mathrm{mV}$, the feedback resistor of this circuit was chosen slightly lower than the lowest measurable impedance. This causes the OPAMP circuit to always attenuate voltages, thus keeping the voltage received by the microphone input below $42 \mathrm{mV}$. As biosensors typically operate in an impedance range of $100 \Omega$ to $100 \mathrm{k} \Omega$ [12], a feedback resistor of $100 \Omega$ was chosen. Due to the 16 bit resolution of the microphone input it should be theoretically possible to measure impedances up to $100 \mathrm{k} \Omega$ with less than $1 \%$ deviation. The system could easily be modified for applications within other impedance ranges by changing this feedback resistor, though the impedance range will remain limited to three decades due to the resolution of the smartphone's audio hardware.

An impedance matching circuit is required for the smartphone to detect the circuit as an external microphone. This required a resistor in the range of $1 \mathrm{k} \Omega$ to $100 \mathrm{k} \Omega$ to be attached parallel to the microphone input. Furthermore, DC voltages were measured at this input. A $10 \mu \mathrm{F}$ capacitor 


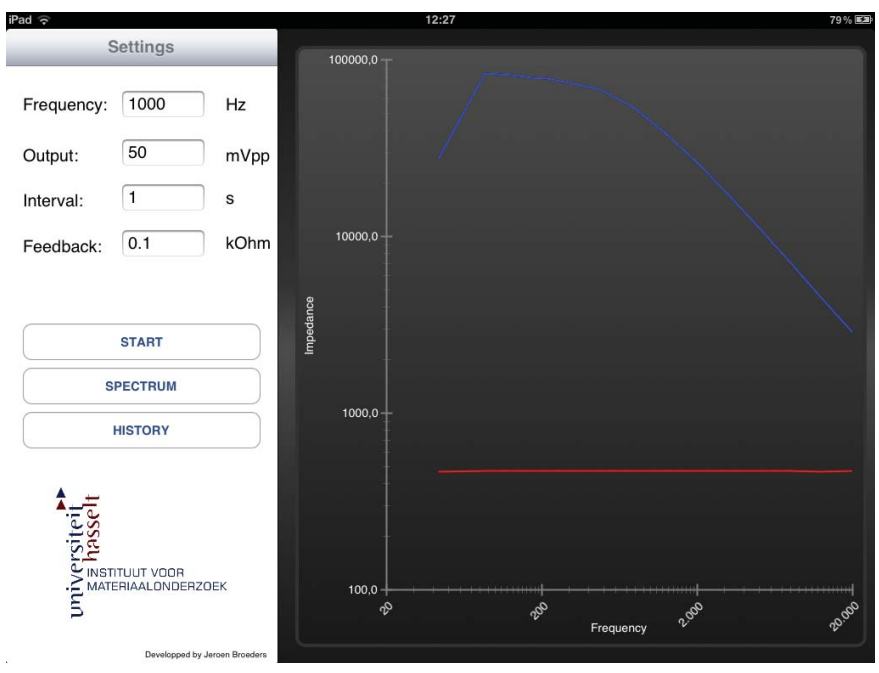

Fig. 2. Screenshot of the software running on an iPad tablet PC.

was used to avoid influence of unwanted voltages coming from the smartphone on the measurement.

Power is supplied to the circuit via two $3 \mathrm{~V}, 140 \mathrm{mAh}$ CR1632 batteries (not shown in the circuit diagram). The operating time was rated at about $70 \mathrm{hrs}$. Although the system is fully battery operated, some noise reduction measures were taken. A four layer PCB layout was chosen consisting of 2 signal layers and a power and ground plane to allow for proper power to ground decoupling. Small package $100 \mathrm{nF}$ and $10 \mu \mathrm{F}$ decoupling capacitors are placed as close as possible to the OPAMP supply pins. The analog measurement ground, indicated by GND in Fig. 1, is connected to the digital audio ground, DGND, directly underneath the audio connector.

\section{Software Methodology}

All software runs on the smartphone/tablet PC used for the measurements in the form of an app. This app was developed by means of Apple Inc.'s Xcode software development kit (SDK), using the proprietary objective-C based Cocoa Touch application programming interface (API). Fig. 2 shows a screenshot of the presented application running on an Apple iPad device. This section discusses the measurement routine while briefly addressing the frameworks and methods used in development of the software.

As a first step in the measurement routine a Bode plot is measured for both channels. An array of frequencies, logarithmically divided between $20 \mathrm{~Hz}$ and $20 \mathrm{kHz}$ is created, after which each frequency is separately generated at the audio output at a fixed peak-to-peak amplitude of $50 \mathrm{mV}$. A Fast Fourier Transform (FFT) is subsequently performed on the values in the microphone input buffer to determine the input amplitude at the current output frequency. Impedance magnitude is calculated from the ratio of the measured input amplitude, the fixed output amplitude and the known feedback resistor of the $I-V$ conversion OPAMP discussed in the previous section.

All audio frameworks within the Cocoa Touch API are included in the Core Audio infrastructure. Since the app will be handling low-level audio data, i.e. outputting and reading sine waves with specified amplitude and frequency, the choice was made to implement Audio Unit plugins, the lowest level of audio framework available. A methodology was chosen where a single unit is responsible for both the output of samples as reading the buffers of input data. To do so, two different callback structures are used which run quasi-simultaneously [20]. An output callback fills the output buffers of both channels with the desired amplitude value for each sample, directly using the following equation:

$$
A_{n}=\sin \left(\frac{n \times 2 \times \pi \times f}{44100}\right) \text {. }
$$

Here $A_{n}$ is the amplitude for sample $n$, with $f$ being the frequency of the output sine wave. The sample rate is fixed at $44.1 \mathrm{kHz}$. The input callback fills a 2048 byte buffer with 102416 bit values, being $23 \mathrm{~ms}$ of audio input data. This audio data is subsequently converted to floating point numbers between 0 and 1 by dividing each sample by 32768 . To allow for stabilization of the excitation signal when measuring electrochemical biosensor measurement cells, a delay of $100 \mathrm{~ms}$ is implemented between signal output and buffer readout.

The vDSP API, contained within the Accelerate framework, provides methods for performing Fourier transforms. A Hanning window is applied to the sample data, after which the data is converted to split-complex format, as is required by the build-in FFT functions. A 1024-point FFT is then performed.

After the measurement of the Bode plots is completed the ideal frequency for time-resolved measurement and plotting is calculated. Measurements are performed on a test strip, coated with the sensing layer, on which liquid analyte is placed. This test strip thus behaves, in essence as an electrochemical cell [21]. Maximum impedimetric response upon target molecule detection in this type of measurement setup can be commonly seen in the capacitive interface between solid sensor layer and analyte [17]. The frequency at which this capacitance is most dominantly present is calculated using the slope of the magnitude plots. The magnitude of the frequency with maximum slope is subsequently measured continuously at intervals of $1 \mathrm{~s}$ to monitor response of the sensor.

The choice was made to implement the open-source Core Plot framework for relatively easy plotting of the time-resolved measurements in a graphically attractive manner. Users have full control over the display of measurements due to the use of scrolling and zooming mechanisms.

\section{Sensor Strip Preparation}

Simplistic biosensor test strips were fabricated as proof of principle, based on the design of commercial glucose sensor strips. A semi-automated Isimat 1000P screen-printing machine was used to print three lines of Dupont 5064 conductive silver ink on a poly(ethylene terephthalate) (PET) foil at an interspacing of $0.8 \mathrm{~mm}$. The resulting electrodes, which measure $2 \mathrm{~mm}$ by $20 \mathrm{~mm}$ in size and are approximately $100 \mu \mathrm{m}$ thick, have a resistivity of about $1 \Omega$. The PET foil containing the electrodes was glued to a $3 \mathrm{~mm}$ thick Teflon strip. A layer of about $100 \mathrm{~nm}$ of Poly(p-phenylene vinylene) (PPV) was spincoated on the electrode strip. 


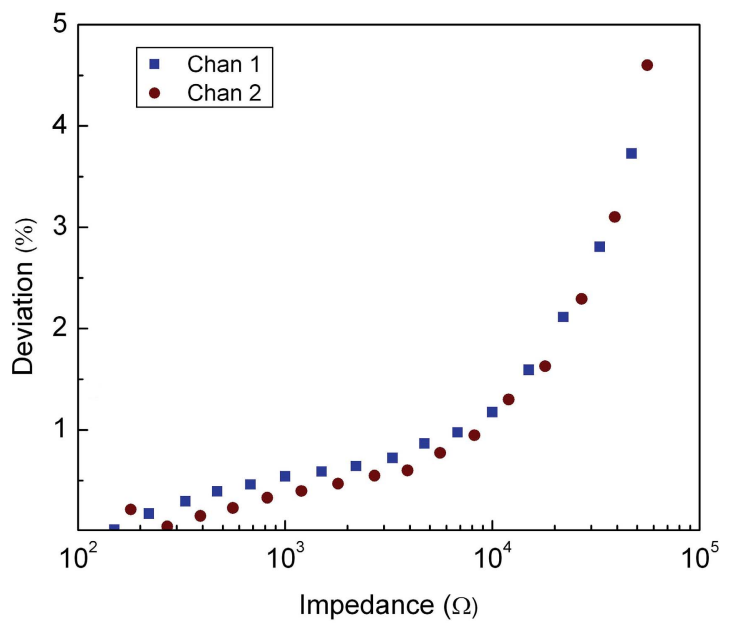

Fig. 3. Accuracy measured on a set of resistors.

Histamine was chosen as the target molecule for the evaluation of this prototype system, primarily due to its easy availability. MIPs suited for detection of this molecule were prepared from a mixture of methacrylic acid $(17.8 \mathrm{mmol})$, ethylene glycol dimethacrylate $(36 \mathrm{mmol})$ and azobisisobutyronitrile $(0.60 \mathrm{mmol})$, together with the template molecule histamine $(8.99 \mathrm{mmol})$, following the protocol described in [13]. Nonimprinted polymers (NIPs), used as a reference, were prepared following the same protocol, though without addition of the template molecule. The MIP and NIP particles were stamped onto the two outer PPV-covered electrodes and subsequently embedded into the PPV layer using a hotplate at $120{ }^{\circ} \mathrm{C}$. Using this manual sensor production technique, a typical surface coverage in between 37 to $39 \%$ is achieved [13]. The center electrode remained covered solely with PPV and is used as a common counter electrode. A $2 \mathrm{~mm}$ thick O-ring with diameter of $7.3 \mathrm{~mm}$ was attached on the electrode strip by means of vacuum grease and functions as a liquid reservoir.

\section{System Evaluation}

System accuracy was analyzed by measuring a set of metal-film resistors from the E12 series within the range of $100 \Omega$ to $100 \mathrm{k} \Omega$. Measurements were performed at a fixed frequency of $2 \mathrm{kHz}$, thus in the center of the audio spectrum. Deviation was calculated in respect to the values measured with a Keithley 2000 digital tabletop multimeter. Fig. 3 shows the deviation in respect to the resistor value for the two measurement channels on the setup.

The lowest measurable resistivity is about $120 \Omega$, corresponding to a microphone input voltage of $42 \mathrm{mV}$, as calculated in the previous sections. Values in between this minimum and a value of $10 \mathrm{k} \Omega$ have a deviation below $1 \%$. The deviation at higher resistivity increases however significantly, reaching 5\% at the aimed maximum impedance of $100 \mathrm{k} \Omega$. This is most likely due to decreasing signal-tonoise ratio, thus lowering the accuracy achieved in the FFT. At a resistivity of $100 \mathrm{k} \Omega$ the $50 \mathrm{mV}$ output signal results in a current of $500 \mathrm{nA}$, making the signal sensitive to noise.

A difference in deviation of about $0.15 \%$ can also be seen between the two channels. This is most likely due to losses

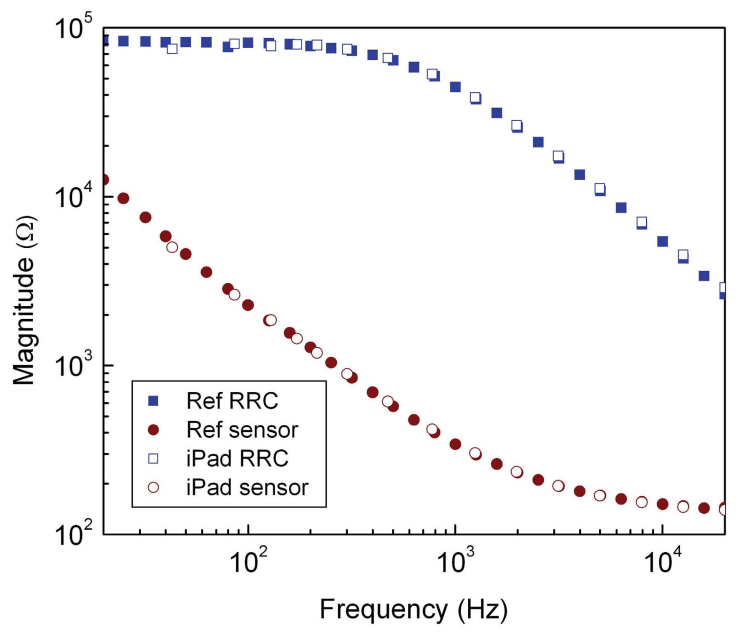

Fig. 4. Magnitude plot for a MIP sensor cell and equivalent randles circuit $[\mathrm{R}(\mathrm{RC})]$ of a diamond-based sensor in respect to a reference device.

in PCB traces, as the shielded cables and connectors were identical for both channels.

As a next step in performance analysis a lab-based biomimetic sensor cell was tested. The sensor layout consists of a sensing layer of histamine MIPs, immobilized on a Ti substrate [4], which is placed in a reservoir containing a liquid analyte. Impedance spectroscopy can be performed by using the substrate as working electrode and a chemically inert conductor, such as a Pt wire, placed in the liquid as counter electrode.

Phosphate buffered saline (PBS), an ionic $\mathrm{pH}$ buffer that emulates biological liquids, was added to the cell as an analyte. A magnitude plot was measured with an iPad tablet PC, using a previously developed analyzer [4] as a reference, and is shown in Fig. 4. Furthermore, the electrical equivalent circuit of a diamond-based DNA sensor setup was constructed. When measuring these electrochemical cells, the substrate and counter electrode as well as the liquid itself can be seen as purely resistive elements. The interface between the sensing layer and liquid behaves like a parallel resistor-capacitor (RC) circuit [21]. This Randles circuit consists of a $3 \mathrm{nF}$ capacitor in parallel with a $82 \mathrm{k} \Omega$ resistor to simulate the interfacial double-layer. A $330 \Omega$ resistor is placed in series to emulate the liquid and electrode resistivity. These measurements show that it is possible to get an adequate indication of the magnitude versus frequency behavior of different biosensor setups. The magnitude data can thus be used to calculate the ideal measurement frequency in software, i.e. the frequency at with the maximum slope is reached and the interfacial capacitance is dominant.

\section{SEnsor Strip ReAdout}

\section{A. Verification in the Lab}

Before performing measurements on the actual MIP-based sensor, the capabilities of the system in combination with the sensor strip were verified. A varying ion concentration of PBS buffer liquid was placed on the test strip. Fig. 5 shows the magnitude plots for three dilutions in demineralized water of 


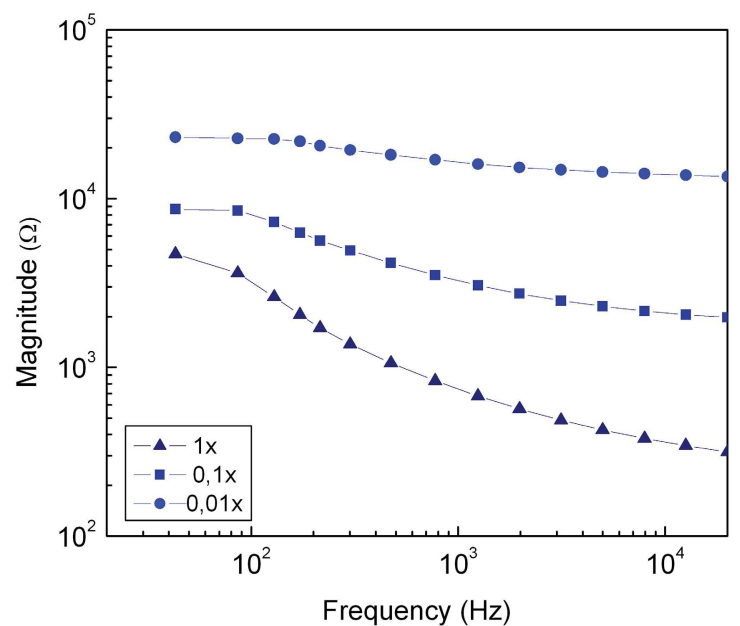

Fig. 5. Magnitude plot for different PBS dilutions on the test strip.

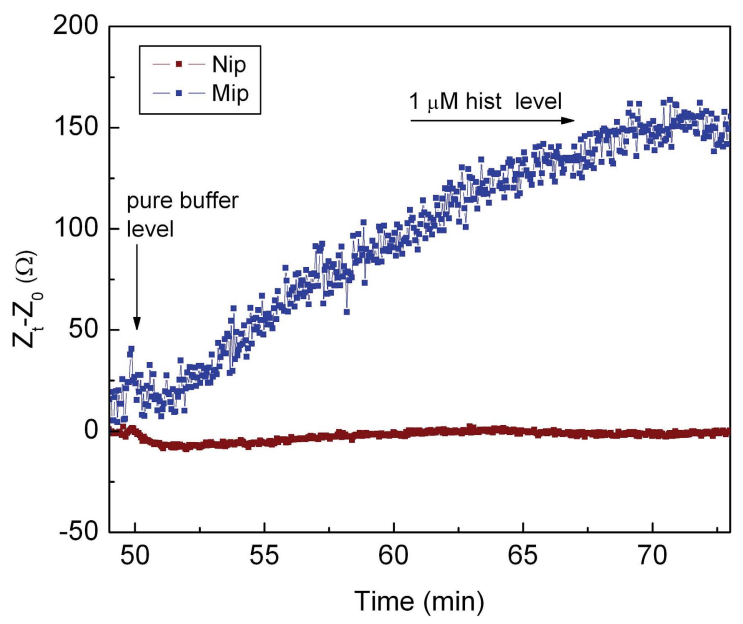

Fig. 6. MIP and NIP magnitude change upon addition of target molecule.

this buffer solution. An increasing magnitude can be seen at lower ion concentrations. These results are consistent with data found in literature [22], indicating correct impedimetric readout of the test strips in liquid media.

As a last step in system evaluation the actual MIP sensor strip was tested against a $1 \mu \mathrm{M}$ concentration of histamine in a PBS solvent. An amount of $60 \mu \mathrm{l}$ PBS was placed inside the O-ring attached to the test strip, after which the O-ring was covered with a PET-foil to avoid evaporation. Magnitude plots were measured for both the MIP and the NIP electrode, after which the ideal measurement frequency was calculated at $500 \mathrm{~Hz}$. Time resolved measurements were subsequently performed on this optimal frequency. The sensor was allowed to stabilize for 50 minutes before histamine was added up to a total target molecule concentration of $1 \mu \mathrm{M}$. Fig. 6 shows the magnitude change relative to the initial magnitude upon addition of the histamine. An impedance magnitude increase in the MIP sample occurs after this addition, after which the signal again stabilizes. The NIP reference fluctuates slightly, possibly due to sudden temperature changes or liquid mobility caused by manual addition of the histamine liquid. However, the NIP stabilizes again at a value close to the initial value.

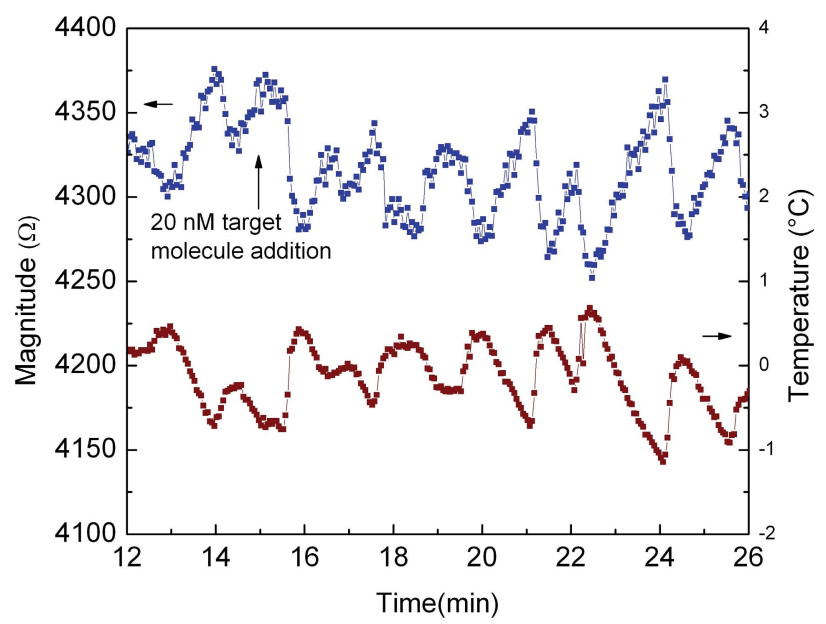

Fig. 7. Impedance magnitude (blue) just before and after histamine addition and induced temperature fluctuations (red).

A magnitude difference of about $150 \Omega$ due to target molecule detection thus occurs in the differential MIP-NIP signal.

\section{B. Toward Home Diagnostic Applications}

When working toward home diagnostics applications, especially when based on hand-held devices, specific design issues come into play. As was addressed in the previous paragraph, the test strips behave as an electrochemical cell. In these cells, impedance fluctuation due to temperature noise is an important issue which can hinder accurate readout of the sensor response. Furthermore, when using the developed system, thermal fluctuations due to handling and movement of the device can reach values of several degrees centigrade. To demonstrate this issue, the senor strip was exposed to such thermal fluctuations using an external PID-based heating unit. A random generator was used in the PID control to induce temperature noise between $25{ }^{\circ} \mathrm{C}$ and $27{ }^{\circ} \mathrm{C}$ at $30 \mathrm{~s}$ intervals. A Pt100 resistance thermometer was measured together with a sensor strip containing solely MIP particles. Fig. 7 shows the effect of the measured temperature fluctuations in respect to the average value of $26{ }^{\circ} \mathrm{C}$ (red), together with the measured impedance magnitude of the MIP sensor (blue). This measured magnitude behaves inversely proportional to the temperature changes, while the addition of a low concentration of $20 \mathrm{nM}$ histamine seems undetectable.

The temperature noise was calculated to be $-70 \Omega /{ }^{\circ} \mathrm{C}$. It should thus be noted that even at relatively high concentrations of target molecules, as is the case in Fig. 6, temperature fluctuations of several degrees would significantly raise the detection limit.

Since the effect of the temperature noise on the impedance can however be easily quantified, it is possible to filter these fluctuations in the measurement software using a reference temperature sensor or the NIP sensor. The latter is already commonly used as a reference to rule out other, much less significant environmental noise sources such as vibrations.

Fig. 8 shows the same magnitude signals as shown in Fig. 7 , though with impedance fluctuations removed using 


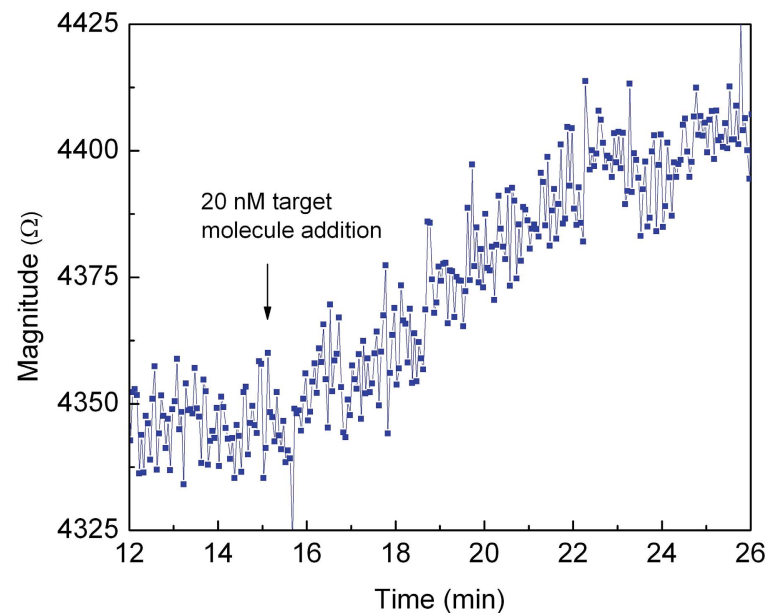

Fig. 8. Impedance magnitude with temperature noise and drift removed.

the calculated thermal noise/impedance ratio. The low concentration of $20 \mathrm{nM}$ of target molecule can now be clearly detected. Furthermore, the remaining noise level lies at about $20 \Omega$, being similar to the noise present in the MIP signal of Fig. 6. The total response at this concentration is about $50 \Omega$, thus well above the present noise level. Furthermore, basic algorithms, such as a moving average filter, can easily be implemented to further reduce this noise if deemed necessary for specific applications.

Another important issue when using the system for home diagnostics is user-induced errors. In theory this setup can be used to measure a wide range of MIP sensors, suited for detection of various target molecules. In order to do so, the user should be able to deposit actual biological samples on the test strip and not just laboratory solutions of the intended target molecule in a buffer solution. Previous research has already shown applications where target molecules could be detected in saliva [23] and blood plasma using MIP based sensors [17]. Furthermore, the amount of liquid used in the discussed measurements is as low as $60 \mu 1$. Since these sensors are concentration-based and within certain practical limits volume-independent, liquid deposition by the user can be simplified in further development toward the level of glucose meters and pregnancy tests.

\section{Conclusion}

A novel way of biosensor readout, using the on-board audio hardware of smartphones and tablet PCs was presented. The required external hardware is kept to a minimum to reduce cost and maintain portability, while intuitive user control and automated data processing are possible by means of an app. Biosensor test strips can be mounted into the device. Small interface modifications in the app can result in a simple to understand true/false indication of specific target molecule concentrations present in a biological liquid, similar to the well-known pregnancy tests.

Characterization on passive components indicates an accuracy of $1 \%$ within the $120 \Omega$ to $10 \mathrm{k} \Omega$ range. Higher impedances are measured with deviations in the range of several percent. In a lab setting biomimetic sensors are commonly read out using commercial electrochemical impedance spectroscopy units such as the Ivium Technologies IviumStat and CompactStat [17], [24], [25]. These devices typically achieve deviations well below $1 \%$ on frequency spectra of 10 decades. However, as was shown in the biomimetic sensor measurements, the resolution of the presented system is high enough to clearly distinguish impedance changes due to binding in the receptor layer of the sensor and the audio frequency spectrum is well suited for readout of the MIP sensor strip. Furthermore, possible measurement noise introduced when using the application outside a lab setting can be easily resolved due to the differential measurement nature. Since these sensors have already been used with success on body fluids the developed system could prove extremely useful in point-of-care settings, where the cost benefit becomes an important factor. The number of smartphone users keeps steadily increasing and the minimal additional hardware represents a minor cost compared to alternative standalone commercial sensor products, e.g. glucose sensors and pregnancy tests. The intuitive app-based user control features add to the relevance of the system in possible commercial settings.

Future work will include the further development and standardization of the test strips and verification of both the sensor strip designs as well as the presented readout technique on different types of biosensors. Furthermore, the MIP sensors used in this paper can already be synthesized for numerous target molecules. Research will point out target molecules of interest for use in home-monitoring applications.

\section{ACKNOWLEDGMENT}

The authors would like to express their gratitude toward L. De Winter, J. Mertens, and J. Baccus for technical support and stimulating discussion.

\section{REFERENCES}

[1] G. M. Schrier, R. G. Moses, I. E. T. Gan, and S. C. Blair, "An evaluation and comparison of Reflolux II and Glucometer II, two new portable reflectancemeters for capillary blood glucose determination," Diabetes Res. Clinical Pract., vol. 4, no. 3, pp. 177-181, Feb. 1988.

[2] J. G. Guan, Y. Q. Miao, and Q. J. Zhang, "Impedimetric biosensors," J. Biosci. Bioeng., vol. 97, no. 4, pp. 219-226, 2004.

[3] M. I. Prodromidis, "Impedimetric immunosensors-A review," Electrochimica Acta, vol. 55, no. 14, pp. 4227-4233, 2010.

[4] J. Broeders, D. Croux, A. Weustenraed, W. Vanaken, S. Duchateau, T. J. Cleij, P. Wagner, R. Thoelen, and W. De Ceuninck, "Embedded unit for point-of-care impedimetric biosensor readout," in Proc. IEEE 14th Int. Conf. High Power Comput. Commun., Jan. 2012, pp. $1571-1577$.

[5] P. Weyner, "Monitoring your health with mobile devices," The New York Times, vol. 162, p. B7, Feb. 2012.

[6] M. Knight. (2011, Oct. 24). Smartphone Detects Danger in a Heartbeat [Online]. Available: http://edition.cnn.com/2011/10/24/tech/ mobile/heart-monitor-smartphone-app/index.html

[7] G. Wulff, "The role of binding site interactions in the molecular imprinting of polymers," Trends Biotechnol., vol. 11, no. 3, pp. 85-87, Mar. 1993.

[8] R. Arshady and K. Mosbach, "Synthesis of substrate-selective polymer by host-guest polymerization," Chem. Phys., vol. 182, no. 2, pp. 687-692, 1981.

[9] P. K. Owens, L. Karlsson, E. S. M. Lutz, and L. I. Andersson, "Molecular imprinting for bio- and pharmaceutical analysis," Trends Anal. Chem., vol. 18, no. 3, pp. 146-154, Mar. 1999. 
[10] S. A. Piletsky, S. Alcock, and A. P. F. Turner, "Molecular imprinting: At the edge of the third millennium," Trends Biotechnol., vol. 19, no. 1, pp. 9-12, Jan. 2001.

[11] B. Sellergren and C. J. Allender, "Molecularly imprinted polymers: A bridge to advanced drug delivery," Adv. Drug Del. Rev., vol. 57, no. 12, pp. 1733-1741, Dec. 2005.

[12] R. Thoelen, R. Vansweevelt, J. Duchateau, F. Horemans, J. D'Haen, L. Lutzen, D. Vanderzande, M. Ameloot, M. vandeVen, T. J. Cleij, and P. Wagner, "A MIP-based impedimetric sensor for the detection of lowMW molecules," Biosensors Bioelectron., vol. 23, no. 6, pp. 913-918, Jan. 2008.

[13] F. Horemans, J. Alenus, E. Bongaers, A. Weustenraed, R. Thoelen, J. Duchateau, L. Lutsen, D. Vanderzande, P. Wagner, and T. J. Cleij, "MIP-based sensor platforms for the detection of histamine in the nanoand micromolar range in aqueous media," Sens. Actuators B, vol. 148 , pp. 392-398, Jul. 2010.

[14] D. Croux, A. Weustenraed, P. Pobedinskas, F. Horemans, H. Diliën, K. Haenen, T. Cleij, P. Wagner, R. Thoelen, and W. De Ceuninck, "Development of multichannel quartz crystal microbalances for MIPbased biosensing," Phys. Status Solidi A, vol. 209, no. 5, pp. 892-899, May 2012.

[15] S. S. A. Pietrzyk, W. Kutner, R. Chitta, M. E. Zandler, and F. D'Souza, "Molecularly imprinted polymer (MIP) based piezoelectric microgravimetry chemosensor for selective determination of adenine," Biosensors Bioelectron., vol. 25, no. 11, pp. 2522-2529, Jul. 2010

[16] P. Cooreman, R. Thoelen, J. Manca, M. vandeVen, V. Vermeeren, L. Michiels, M. Ameloot, and P. Wagner, "Impedimetric imunosensors based on the conjugated polymer PPV," Biosensors Bioelectron., vol. 20, no. 10 , pp. 2151-2161, Apr. 2005.

[17] M. Peeters, F. J. Troost, B. van Grinsven, F. Horemans, J. Alenus, M. S. Murib, D. Keszthelyi, A. Ethirajan, R. Thoelen, T. J. Cleij, and P. Wagner, "MIP based biomimetic sensor for the electronic detection of serotonine in human blood plasma," Sens. Actuators B, vols. 171-172, pp. 602-610, Aug.-Sep. 2012.

[18] B. van Grinsven, N. Vanden Bon, L. Grieten, M. Murib, S. D. Janssens, K. Haenen, E. Schneider, S. Ingebrandt, M. J. Schoning, V. Vermeeren, M. Ameloot, L. Michiels, R. Thoelen, W. De Ceuninck, and P. Wagner, "Rapid assessment of the stability of DNA duplexed by impedimetric real-time monitoring of chemical induced denaturation," Lab Chip, vol. 11, pp. 1656-1663, Mar. 2011.

[19] C. H. Fry and R. I. Jabr, "The action potential and nervous conditions," Surgery, vol. 28, no. 2, pp. 49-54, Feb. 2010.

[20] M. Tyson. (2008, Nov. 4). Using RemoteIO Audio Unit [Online]. Available: http://atastypixel.com/blog/using-remoteio-audio-unit/

[21] Gamry Instruments Inc. (2010). Basics of Electrochemical Impedance Spectroscopy, Warminster, PA, USA [Online]. Available: http://www.gamry.com/assets/Application-Notes/Basics-of-EIS.pdf

[22] B. van Grinsven, T. Vandenryt, S. Duchateau, A. Gaulke, L. Grieten, R. Thoelen, S. Ingebrandt, W. De Ceuninck, and P. Wagner, "Customized impedance spectroscopy device as possible sensor platform for biosensor applications," Phys. Status Solidi A, vol. 207, no. 4, pp. 919-923, Apr. 2010.

[23] M. H. Lee, J. L. Thomas, H. Y. Tseng, W. C. Lin, B. D. Liu, and H. Y. Lin, "Sensing of digestive proteins in saliva with a molecularly imprinted poly(ethylene-co-vinyl alcohol) thin film coated quartz crystal microbalance sensor," Appl. Mater. Inter., vol. 3, no. 8, pp. 3064-3071, Jul. 2011.

[24] R. M. Santos, M. S. Rodrigues, J. Laranjinha, and R. M. Barbosa, "Biomimetic sensor based on hemin/carbon nanotubes/chitosan modified microelectrode for nitric oxide measurement in the brain," Biosensors Bioelectron., vol. 44, pp. 152-159, Jul. 2013.

[25] C. Martensson and V. A. Hernandez, "Ubiquinone-10 in goldimmobilized lipid membrane structures acts as a sensor for acetylcholine and other tetraalkylammonium cations," Bioelectrochemistry, vol. 88, pp. 171-180, Dec. 2012.

Jeroen Broeders received the M.S. degree in electronics-ICT from Xios University College, Diepenbeek, Belgium, in 2009. He is currently pursuing the $\mathrm{Ph} . \mathrm{D}$. degree in industrial engineering sciences at the Institute for Materials Research, Hasselt University, Hasselt, Belgium, where he is working on the development of readout techniques for biosensors.
Dieter Croux received the M.S. degree in electrical engineering from KU Leuven, Leuven, Belgium, in 2008. He is currently pursuing the Ph.D. degree in physics at the Institute for Material Research, Hasselt University, Hasselt, Belgium. His current research interests include the development of readout techniques and sensor platforms for small molecule detection through imprinted polymers.

Marloes Peeters received the M.S. degree in polymer chemistry from Eindhoven University of Technology, Eindhoven, The Netherlands, in 2009. She is currently pursuing the Ph.D. degree at the Institute for Material Research, Hasselt University, Hasselt, Belgium. She is currently working on the in vitro detection of small molecules based on polymer-type immobilization platforms.

Thomas Beyens received the B.S. degree in electronics-ICT in 2011 and is currently pursuing the M.S. degree at Xios University College, Diepenbeek, Belgium, and intern at the Institute for Materials Research, Hasselt University, Hasselt, Belgium.

Stijn Duchateau received the M.S. degree in electronics-ICT from Xios University College, Diepenbeek, Belgium, in 2008. He is currently pursuing the $\mathrm{Ph} . \mathrm{D}$. degree at the Institute for Materials Research, Hasselt University, Hasselt, Belgium. He is a Teaching Assistant with Xios University College.

Thomas J. Ceij received the Ph.D. degree in polymeric materials science from Utrecht University, Utrecht, The Netherlands, in 1999. After a stay of three years in the U.S. as an Assistant Professor with Louisiana State University, Baton Rouge, LA, USA, he was appointed as a Full Professor of organic and bio-polymer chemistry with the Institute for Material Research, Hasselt University, Hasselt, Belgium. His expertise at the Laboratory for Organic and Bio-Polymer Chemistry focuses on the design, synthesis, characterization and application of functional polymeric materials. State of the art facilities for polymer synthesis and characterization are available.

Prof. Cleij has published over 50 papers on polymeric materials science and engineering.

Patrick Wagner received the Ph.D. degree in experimental solid-state physics with a focus on cuprate superconductors from Technical University Darmstadt, Darmstadt, Germany, in 1994. From 1995 to 2001, he was a Post-Doctoral Researcher with the Laboratory of Solid State Physics and Magnetism, Catholic University Leuven, Leuven, Belgium, where he studied the magnetotransport properties of mixed-valency magnetic oxides. Since 2001, he has been a Professor of physics with Hasselt University, Hasselt, Belgium, and is responsible for the development of label-free readout techniques for DNA-, protein-, and small molecule sensors.

Prof. Wagner is a recipient of the WE Heraeus Award, the Marie-Curie Fellowship of the European Union, the Methusalem Grant of the Flemish Government and is past President of the Belgian Physical Society. He has published over 150 papers in literature.

Ronald Thoelen received the Master of Science degree in applied physics from the Eindhoven University of Technology, Eindhoven, The Netherlands. In 2008, he received the Ph.D. degree in biosensors from Hasselt University, Hasselt, Belgium. Since 2008, he has been a Professor with the Faculty of Engineering Technology, Electronics-ICT, Xios University College, Diepenbeek, Belgium. He coordinates the research of embedded biosensor development.

Ward De Ceuninck received the Ph.D. degree in experimental physics with a focus on the relaxation kinetics of amorphous alloys from Limburgs Universitair Centrum, Limburgs, Belgium, in 1990. Currently, he is a Professor with Hasselt University, Hasselt, Belgium, and is leading a research group with the Institute of Materials Research and is involved in the development of high precision electrical readout schemes for biosensors.

Prof. De Ceuninck has been involved in reliability of microelectronics and has published more than 100 papers in literature and is the owner of more than 10 patents in the field of reliability and biosensors. 